\title{
Distribuição espacial e temporal da raiva canina e felina em Minas Gerais, 2000 a 2006
}

[Spatial and temporal distribution of canine and feline rabies in Minas Gerais, Brazil, from 2000 to 2006]

\author{
A.D. Barbosa ${ }^{1,4}$, J.A. Silva ${ }^{2 *}$, E.C. Moreira ${ }^{2}$, J.N.C. Meneses ${ }^{2}$, D.F. Magalhães ${ }^{3}$, \\ F.L. Menezes ${ }^{1,4}$, C.S.F. Oliveira ${ }^{1,4}$ \\ ${ }^{1}$ Aluna de graduação - EV-UFMG - Belo Horizonte, MG \\ ${ }^{2}$ Escola de Veterinária - UFMG \\ Caixa Postal 567 \\ 30123-970 - Belo Horizonte, MG \\ ${ }^{3}$ Aluna de pós-graduação - EV-UFMG - Belo Horizonte, MG \\ ${ }^{4}$ Bolsista de Iniciação Científica
}

\begin{abstract}
RESUMO
Estudou-se a epidemiologia da raiva em cães e gatos, em Minas Gerais, e realizou-se um estudo descritivo de seu comportamento, de 2000 a 2006. Utilizaram-se, como fonte de dados, os relatórios mensais dos laboratórios do Centro de Controle de Zoonoses de Belo Horizonte e do Instituto Mineiro de Agropecuária. Os resultados demonstraram positividade de $1,5 \%$ para raiva canina e $0,7 \%$ para raiva felina. Houve tendência ao decréscimo do número de casos positivos de raiva canina $(\mathrm{y}=-3,2143 \mathrm{x}+$ 19,714) e também para a raiva felina, com apenas dois casos, ambos em 2000. As regiões com diagnóstico positivo coincidiram com as de menor desenvolvimento sócioeconômico.
\end{abstract}

Palavras-chave: cão, gato, raiva, distribuição espaço-temporal, epidemiologia

\begin{abstract}
In order to know the epidemiology of rabies in dogs and cats, in Minas Gerais State, a descriptive study of its behavior was carried out from 2000 to 2006. Data of monthly reports colleted at the laboratories of "Centro de Controle de Zoonoses (CCZ)" from Belo Horizonte" and "Instituto Mineiro de Agropecuária (IMA)" were used. Positivity of $1.50 \%$ for canine rabies and $0.70 \%$ for feline rabies were observed. A tendency to the decrease of canine rabies positive cases was found $(y=-3.2143 x+19.714)$ and the same was observed for cats, since only two cases occurred, both in 2000. The regions that present positive diagnostics also showed the lowest social economic development.
\end{abstract}

Keywords: dog, cat, rabies, spatial temporal distribution, epidemiology

\section{INTRODUÇÃO}

O Programa de Profilaxia da Raiva Urbana, elaborado em 1969, coordenado pelo Ministério da Saúde, teve como prioridade implantar políticas de atuação pública. A primeira foi garantir o acesso de todas as pessoas, agredidas por animais e consideradas expostas ao risco da raiva, ao tratamento anti-rábico com vacina e soro de qualidade. Simultaneamente, construíram-se laboratórios para o diagnóstico rápido, com o uso das técnicas de imunofluorescência direta e inoculação em camundongos. $O$ trabalho de educação sanitária visando à ampla participação da sociedade no controle foi outro ponto considerado importante (Norma..., 1995). Essas ações surtiram efeito, e a expectativa era de se conseguir a erradicação da raiva canina e felina até 2000 .

O êxito pleno dessa meta foi alcançado nos estados de São Paulo, Santa Catarina, Rio Grande do Sul e Rio de Janeiro. Os estados de Minas Gerais, Goiás, Mato Grosso, Mato Grosso do Sul e Pernambuco conseguiram erradicar a raiva canina e felina em $90 \%$ de seus municípios.

Recebido em 10 de junho de 2007

Aceito em 26 de maio de 2008

*Autor para correspondência (corresponding author)

E-mail: jasilva@vet.ufmg.br 
Em Minas Gerais, Belo Horizonte registrou em 1989 seu último caso canino e os 35 municípios da região metropolitana, a partir de 1995, também conseguiram zerar a raiva canina, felina e humana (Brito e Chamone, 2002).

No período de 1991 a 1999, em Minas Gerais, foram registrados 155 casos de raiva canina; desses, 147 foram de cães das regiões norte e nordeste, 11 casos de raiva felina e 33 casos de raiva humana (Miranda et al., 2003).

A transmissão e o endemismo da raiva canina e felina para o homem dependem das relações sociais e econômicas prevalentes em cada região. A densidade da população canina, a adoção de medidas de atenção veterinária, os cuidados com a posse e os tipos de confinamento animal estão associados ao nível cultural e social de seus proprietários. Minas Gerais possui áreas com significativos níveis de baixo desenvolvimento social, fato que reflete nos padrões de saúde e da ocorrência de riscos para doenças que a sociedade moderna conseguiu erradicar (Minas..., 2002).

O objetivo deste trabalho foi conhecer a distribuição temporal e espacial da raiva canina e felina em Minas Gerais, de 2000 a 2006.

\section{MATERIAL E MÉTODOS}

A área de estudo foi o estado de Minas Gerais, que está situado na região Sudeste do Brasil e possui 853 municípios em $588.383,6 \mathrm{~km}^{2}$, o que equivale a $6,9 \%$ do território nacional. A população humana, em 2007, era de 19.273.506 (Contagem..., 2007). Trinta por cento da população do estado encontrava-se abaixo da linha de pobreza, e o índice de desenvolvimento humano (IDH) era de 0,773 , considerado, portanto, como área de médio desenvolvimento (Oliveira, 2005).

O estudo descritivo do comportamento da raiva canina e felina em Minas Gerais, de 2000 a 2006, foi realizado segundo variáveis relativas à espécie animal, lugar e tempo. Utilizaram-se fontes de dados secundários mensais procedentes dos laboratórios do Centro de Controle de Zoonoses (CCZ) de Belo Horizonte e do Instituto Mineiro de Agropecuária (IMA). De 2002 a 2004, não foram realizados exames pelo $\mathrm{CCZ}$, pois este estava passando por processo de reforma de suas instalações. A utilização de dados dessas instituições se justifica por serem as responsáveis pela maioria dos diagnósticos realizados no estado.
Utilizou-se a técnica de imunofluorescência direta para a realização dos diagnósticos.

Elaborou-se um banco de dados no qual se registraram, para cada exame, o município de origem do animal, a espécie, a instituição realizadora do exame, o diagnóstico, o mês e o ano.

Os dados foram processados no software EPIData 3.1, que geraram informações para análise epidemiológica da raiva canina e felina. A distribuição temporal e espacial da raiva canina e felina foi representada por tabelas e estimativas de tendência construídas com auxilio do programa Excel versão 2003. Para análise e representação da distribuição espacial da doença, utilizou-se o aplicativo de mapeamento Tabwin versão 1.4.

\section{RESULTADOS}

De 2000 a 2006, foram realizados 3.194 exames laboratoriais para diagnóstico de raiva canina. Desses, $2.084(65,2 \%)$ foram feitos pelo $\mathrm{CCZ}$ e $1.110(34,8 \%)$ pelo IMA. Quarenta e oito exames $(1,5 \%)$ apresentaram resultados positivos e 3.146 $(98,5 \%)$ foram negativos. O percentual de positividade foi menor que o de negatividade, e houve redução da porcentagem de amostras positivas de 2000 a 2006. Verificou-se, nesse período, aumento no número de exames e ausência de casos de raiva canina em 2005 e em 2006 (Tab. $1)$.

Em relação à raiva felina, foram realizados 285 exames, sendo 169 (59,3\%) feitos pelo CCZ e 116 (40,7\%) pelo IMA (Tab. 2). A ocorrência de apenas dois casos de raiva em gatos, ambos em 2000, representou $0,7 \%$ do total de exames realizados no período (Tab. 2).

A distribuição espacial da raiva canina e felina foi avaliada, anualmente, nos 853 municípios de Minas Gerais e esteve presente em 17 (Tab. 3). Em Governador Valadares, a positividade foi persistente de 2000 a 2002, e esse foi o município com maior número de casos diagnosticados de raiva. A Fig. 1 mostra a distribuição espacial da doença por município, de 2000 a 2006. Observa-se que o agravo ocorreu nas regiões do Vale do Rio Doce, Vale do Mucuri, Vale do Jequitinhonha (Alto e Médio Jequitinhonha) e Zona da Mata. A raiva felina foi diagnosticada somente em dois municípios, Itinga e São João Evangelista. 
Com relação à freqüência na remessa de amostras para diagnóstico, houve tendência de aumento e, conseqüentemente, aumento do número de exames laboratoriais realizados (Fig. 2).
Quanto ao número de casos de raiva canina, houve tendência ao decréscimo de diagnósticos positivos para a raiva nessa espécie (Fig. 3).

Tabela 1. Exames de imunofluorescência direta para diagnóstico de raiva canina, realizados pelo Centro de Controle de Zoonoses (CCZ) e Instituto Mineiro de Agropecuária (IMA), em Minas Gerais, entre 2000 e 2006

\begin{tabular}{cccccc}
\hline Ano & CCZ & IMA & Negativos & Positivos $(\%)$ & Total $(\%)$ \\
\hline 2000 & 309 & 97 & 391 & $15(3,7)$ & $406(12,7)$ \\
2001 & 320 & 132 & 430 & $22(4,9)$ & $452(14,2)$ \\
2002 & 0 & 159 & 157 & $2(1,3)$ & $159(5,0)$ \\
2003 & 0 & 170 & 162 & $8(4,7)$ & $170(5,3)$ \\
2004 & 0 & 189 & 188 & $1(0,5)$ & $189(5,9)$ \\
2005 & 435 & 323 & 758 & $0(0,0)$ & $758(23,7)$ \\
2006 & 1020 & 40 & 1060 & $0(0,0)$ & $1060(33,2)$ \\
Total & 2084 & 1110 & 3146 & $48(1,5)$ & $3194(100,0)$ \\
\hline
\end{tabular}

Tabela 2. Exames de imunofluorescência direta para diagnóstico da raiva felina, realizados pelo Centro de Controle de Zoonoses (CCZ) e Instituto Mineiro de Agropecuária (IMA), em Minas Gerais, entre 2000 e 2006

\begin{tabular}{cccccc}
\hline Ano & CCZ & IMA & Negativos & Positivos $(\%)$ & Total $(\%)$ \\
\hline 2000 & 36 & 12 & 46 & $2(4,2)$ & $48(16,8)$ \\
2001 & 25 & 16 & 41 & $0(0,0)$ & $41(14,4)$ \\
2002 & 0 & 31 & 31 & $0(0,0)$ & $31(10,9)$ \\
2003 & 0 & 17 & 17 & $0(0,0)$ & $17(6,0)$ \\
2004 & 0 & 17 & 17 & $0(0,0)$ & $17(6,0)$ \\
2005 & 30 & 17 & 47 & $0(0,0)$ & $47(16,5)$ \\
2006 & 78 & 6 & 84 & $0(0,0)$ & $84(29,4)$ \\
Total & 169 & 116 & 283 & $2(0,7)$ & $285(100,0)$ \\
\hline
\end{tabular}

Tabela 3. Municípios positivos para raiva canina em Minas Gerais, entre 2000 e 2006

\begin{tabular}{lcc}
\hline Município & Examinados & Positivos \\
\hline Água Boa & 1 & 1 \\
Águas Formosas & 1 & 1 \\
Araçuaí & 2 & 1 \\
Bias Fortes & 1 & 1 \\
Capelinha & 2 & 1 \\
Coluna & 2 & 2 \\
Frei Lagonegro & 2 & 2 \\
Guanhães & 1 & 1 \\
Governador Valadares & 80 & 17 \\
Materlândia & 1 & 1 \\
Novo Oriente de Minas & 2 & 2 \\
Rio Vermelho & 1 & 1 \\
Sabinópolis & 4 & 2 \\
Santa Maria do Suaçuí & 1 & 1 \\
São João Evangelista & 2 & 2 \\
São Sebastião do Maranhão & 12 & 6 \\
Teófilo Otoni & 15 & 6 \\
\hline
\end{tabular}




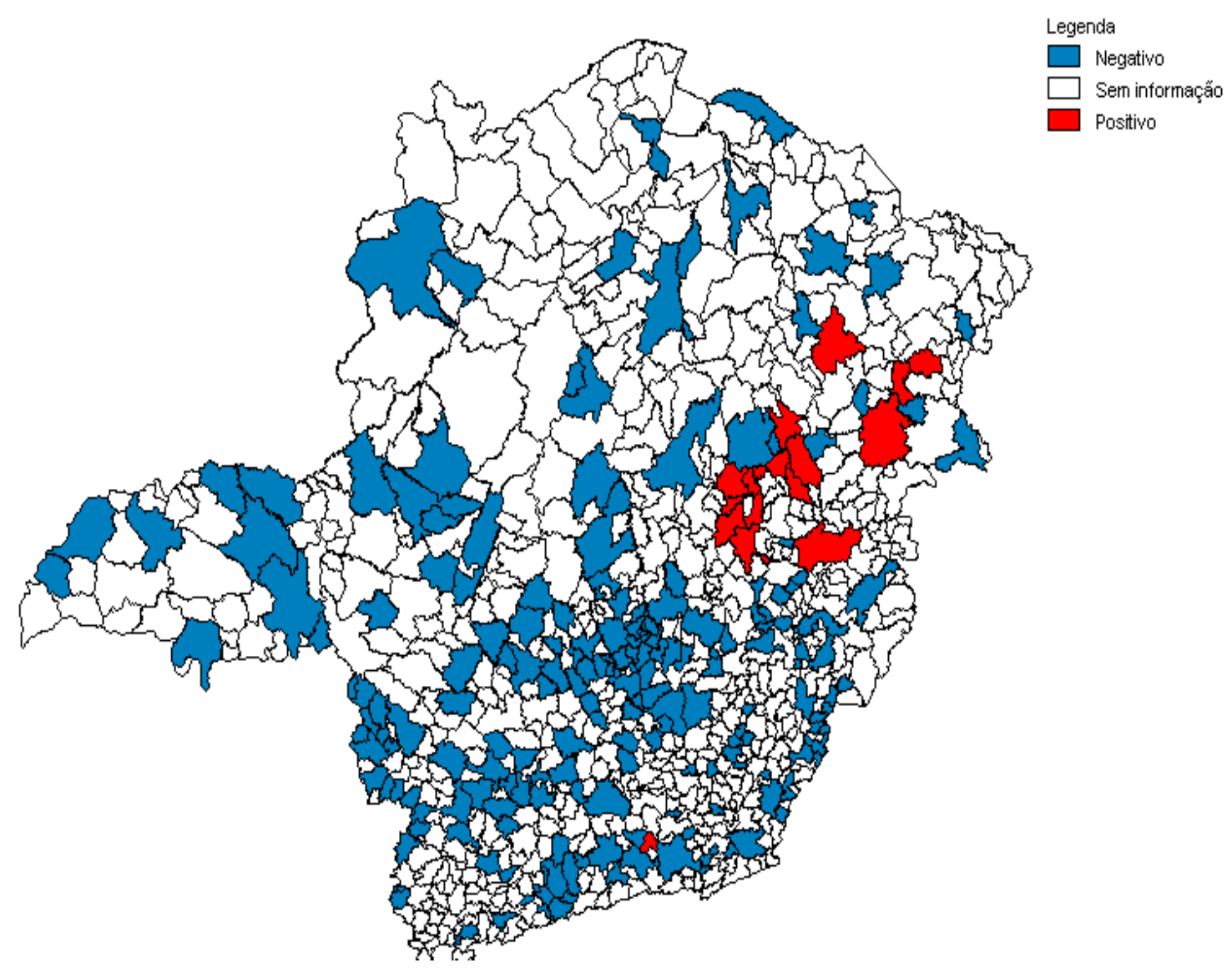

Figura 1. Distribuição da raiva canina nos municípios de Minas Gerais, entre 2000 e 2006.

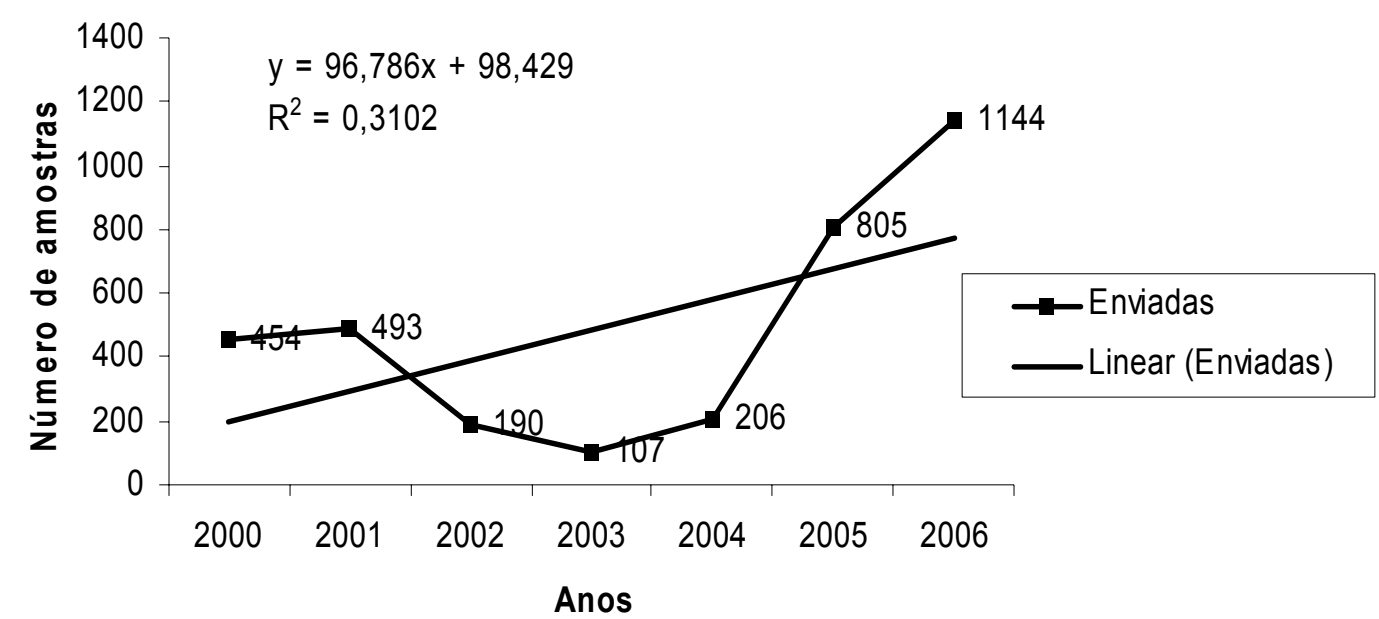

Figura 2. Tendência do envio de amostras para diagnóstico de raiva canina e felina em Minas Gerais, entre 2000 e 2006. 


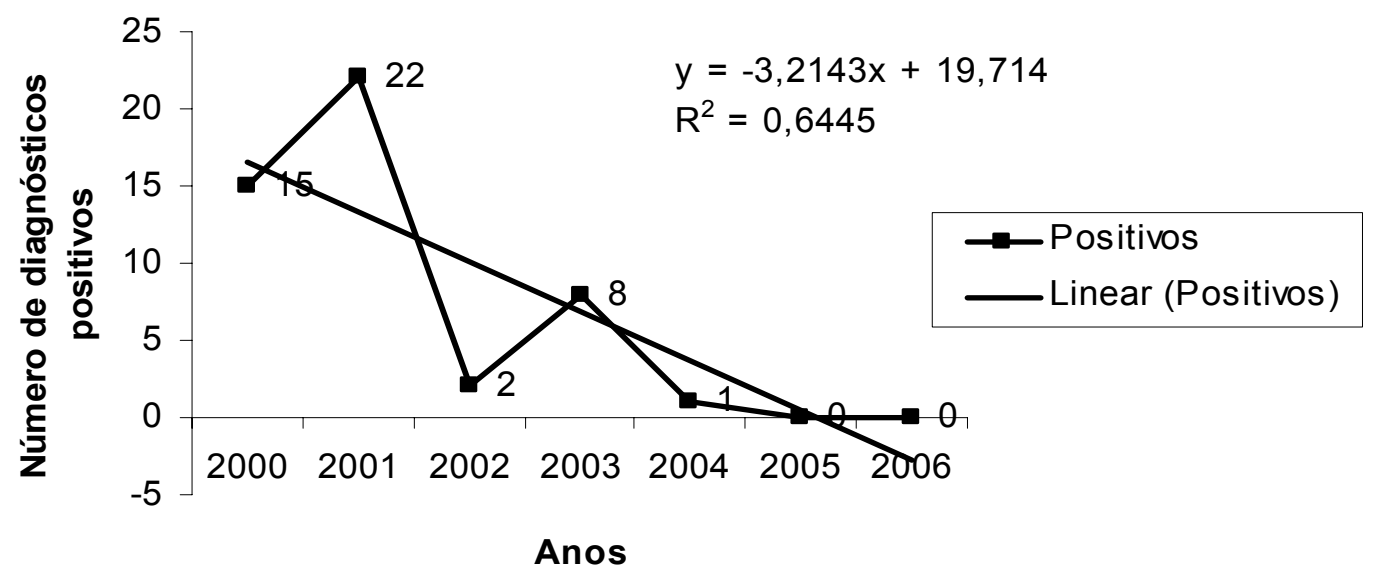

Figura 3. Tendência de diagnósticos positivos para raiva canina em Minas Gerais, entre 2000 e 2006.

\section{DISCUSSÃO E CONCLUSÕES}

Houve tendência ao aumento do número de amostras enviadas para diagnóstico laboratorial de raiva canina e felina em Minas Gerais, de 2000 a 2006, mesmo com acentuada redução desse número de 2002 a 2004, o que pode ser explicado, em parte, pelo processo de reforma do laboratório do CCZ de Belo Horizonte.

No período estudado pôde-se observar tendência à redução de diagnósticos positivos para raiva canina. Esse fato, associado ao aumento do envio de amostras para diagnóstico, permite inferir que houve aplicação de medidas eficazes de controle da doença. Acredita-se que as medidas preconizadas pelo Manual... (1999), tais como vacinação, apreensão de animais errantes, observação clínica dos animais e colheita e envio de amostras para exames de laboratório, além da educação em saúde foram implementadas adequadamente.

Araújo (2000) considerou que a divulgação intensiva das ações de vacinação antes de sua realização influenciava o aumento de coberturas vacinais. Esse autor concluiu que o desencadeamento e o direcionamento do controle são fatores de efetividade para avanço da eliminação da raiva humana no país. Isso pode, portanto, ter contribuído para a realidade observada neste estudo. $\mathrm{O}$ progressivo controle da raiva canina em Minas Gerais leva a uma situação epidemiológica emergente, a qual coloca cada vez mais em evidência a importância da raiva em morcegos e outros animais silvestres, conforme salientaram Lontai (1996) e Belloto (2001), ao considerarem a epidemiologia da raiva na América Latina.

Nos 17 municípios mineiros que apresentaram casos diagnosticados de raiva canina, que coincidem com os de menores índices de desenvolvimento humano do estado, podem ter ocorrido falhas nas ações de controle da doença ou no direcionamento delas. Essas falhas podem incluir falta de controle do trânsito de animais, não apreensão de cães errantes, administração de vacinas em animais imunoincompetentes e baixa cobertura vacinal. A ausência de controle de qualidade de vacinas, nas Diretorias Regionais de Saúde de Diamantina e Governador Valadares foi verificada por Miranda et al. (2003), e esse fato pode estar relacionado à ocorrência de casos de raiva canina nesses municípios. Maior número de casos de raiva canina ocorridos no município de Governador Valadares também foi verificado pelos autores citados.

A distribuição espacial da raiva canina em Minas Gerais mostra que não há correlação entre concentração da população canina e ocorrência da doença, fato já observado por Miranda et al. (2003), no período de 1991 a 1999. O comportamento espacial da doença de 2000 a 2006 confirma a tendência à diminuição dos 
casos de raiva canina na capital e no interior do Estado, fato também observado por Luz (1988). Os resultados do presente estudo diferem da expectativa de Schneider (1990), que concluiu que a Região Metropolitana de Belo Horizonte possuía alto risco para raiva. A dinâmica espaçotemporal liga-se aos momentos distintos do fenômeno raiva e do planejamento das ações de controle.

Segundo Acha e Szyfres (1986), o gato é um hospedeiro acidental do vírus, não desempenhando papel importante no ciclo natural da doença. Os resultados obtidos em relação à raiva felina confirmaram essa afirmação. Esses autores relatam a necessidade da vacinação de felinos domésticos, uma vez que a espécie é importante fonte de infecção humana. Dessa forma, a eficiência da cobertura vacinal em cães e gatos pode ter sido fator de contribuição para o baixo número de casos de raiva felina diagnosticados de 2000 a 2006. Segundo o Manual... (1999), a raiva felina desaparece assim que a doença é controlada na espécie canina. Esse relato poderia justificar a correlação positiva observada entre a raiva em cães e gatos, uma vez que ambas apresentaram tendência à redução de casos diagnosticados no período.

Com base nos resultados obtidos, conclui-se que a ocorrência da raiva canina e felina é heterogênea em Minas Gerais. Os municípios com casos positivos coincidem com as áreas de menor desenvolvimento sócioeconômico do estado, padrão da história natural dessa zoonose. Assim, a elaboração de estratégias diferenciadas para erradicar a raiva nas regiões ainda com situação de risco deverá, em curto prazo, atingir a meta estabelecida para que em 2010 todo o território nacional seja considerado livre para raiva canina e felina.

\section{REFERÊNCIAS BIBLIOGRÁFICAS}

ACHA, P.N.; SZYFRES, B. Zoonosis y enfermedades transmissibles comunes al hombre y a los animales. 2.ed. Washington, DC: OPAS/OMS, 1986. p.502-525.

ARAÚJO, F.A. A situação da raiva no Brasil. In: RAIVA: SEMINÁRIO INTERNACIONAL DA
RAIVA, 1., 2000, São Paulo. Anais... São Paulo: Instituto Pasteur, 2000. p.22. (Resumo).

BELOTTO, A.J. Raiva transmitida por morcegos nas Américas: impacto na saúde pública e na produção. In: SEMINÁRIO INTERNACIONAL MORCEGOS COMO TRANSMISSORES DA RAIVA, 1., 2001. São Paulo. Anais... São Paulo: Instituto Pasteur, 2001.

BRITO, M.G.; CHAMONE,T.L. Ações de controle da raiva canina e felina no estado de Minas Gerais, 1999-2002. Bol. Epidemiol., v.6, p.1-8, 2002.

CONTAGEM da população 2007. Rio de Janeiro: IBGE, 2007. Disponível em: $<$ http:// www.ibge.gov.br./home/estatistica/populacao/co ntagem2007/contagem.pdf.> Acessado em: 15 nov. 2007.

LONTAI, I. Rabies prevention current situation in Southeast and Central Europe. Budapest: National Institute of Public Health, 1996. 77p.

LUZ, C.R. Estudo cronológico sobre a raiva em Minas Gerais - no período de 1976 a 1986. 1988. 122f. Dissertação (Mestrado) - Escola de Veterinária, Universidade Federal de Minas Gerais, Belo Horizonte, MG.

MANUAL de vacinação contra raiva de cães e gatos: informe técnico. 3.ed. São Paulo: Instituto Pasteur, 1999. 31p.

MINAS Gerais do século XXI. Belo Horizonte: Rona Editora, 2002.

MIRANDA, C.F.J.; SILVA, J.A.; MOREIRA, E.C. Raiva humana transmitida por cães: áreas de risco em Minas Gerais, Brasil, 1991-1999. Cad. Saude Public., v.19, p.91-99, 2003.

NORMA técnica de tratamento profilático antirábico humano. 2.ed. Brasília: FUNASA, 1995. 40p.

OLIVEIRA, V.M.R. O atendimento anti-rábico humano em Minas Gerais, de1999- 2004. 2005. 80f. Dissertação (Mestrado) - Escola de Veterinária, Universidade Federal de Minas Gerais, Belo Horizonte, MG.

SCHNEIDER, M.C., ALMEIDA, G.A., SOUZA, L.M., et al. Controle da raiva no Brasil de 1980 a 1990. Rev. Saude Public., v.30, p.196-203, 1990. 